\title{
Gradient estimates for inverse curvature flows in hyperbolic space
}

\begin{abstract}
We prove gradient estimates for hypersurfaces in the hyperbolic space $\mathbb{H}^{n+1}$, expanding by negative powers of a certain class of homogeneous curvature functions $F$. We obtain optimal gradient estimates for hypersurfaces evolving by certain powers $p>1$ of $F^{-1}$ and smooth convergence of the properly rescaled hypersurfaces. In particular, the full convergence result holds for the inverse Gauss curvature flow of surfaces without any further pinching condition besides convexity of the initial hypersurface.
\end{abstract}

Keywords: Curvature flows, Inverse curvature flows, Hyperbolic space

MSC: 35K55, 53C21, 53C44

\section{Introduction and the main result}

This short note is a direct improvement of [4], in which we considered inverse curvature flows in the hyperbolic space $\mathbb{H}^{n+1}, n \geq 2$, of the form

$$
\begin{aligned}
\dot{x} & =\frac{1}{F^{p}} \nu, \quad 0<p<\infty, \\
x(0, M) & =M_{0},
\end{aligned}
$$

where

$$
x:[0, \infty) \times M \hookrightarrow \mathbb{H}^{n+1}
$$

is a family of embeddings, $F$ is a curvature function satisfying some additional properties specified later and $M_{0}$ is a suitable initial hypersurface. We showed that under certain conditions on $F$ and $M_{0}$ this flow exists for all time and converges to a well-defined smooth function after rescaling. In case $p>1$ we had to impose quite restrictive conditions on $F$ and $M_{0}$, namely $F$ was supposed to vanish on the boundary of

$$
\Gamma_{+}=\left\{\left(\kappa_{i}\right) \in \mathbb{R}^{n}: \kappa_{i}>0 \quad \forall i=1, \ldots, n\right\}
$$

and $M_{0}$ had to satisfy a pinching condition on the oscillation, cf. [4, Thm. 1.2, (2)]. This condition was designed to ensure that the gradient of the initial convex hypersurface $M_{0}$ was small. Then it was possible to show that it remained small. The unsatisfactory thing was that we were not able to treat powers of the Gaussian curvature that were larger than $n^{-1}$ without this restriction. The aim of this note is to provide gradient estimates which allow to treat (1) for some powers $1<p \leq p_{0}$, where $p_{0}$ will depend on the curvature function. In case of the Gaussian curvature, $F=n K^{\frac{1}{n}}$, we will obtain $p_{0}=\frac{n}{n-1}$. This includes the full convergence result in case of the inverse Gauss curvature flow for surfaces with arbitrary convex initial hypersurface, a problem which to our knowledge has not been considered in the literature before. The class of curvature functions we are able

^Corresponding Author: Julian Scheuer: Institut für Angewandte Mathematik, Universität Heidelberg, E-mail: scheuer@math.uni-heidelberg.de 
to prove the main result for is closely related to the class $\left(K^{\star}\right)$ defined in [2, Def. 2.2.15]. We will come back to this issue in some more detail later.

Before we state the main result, let us formulate the assumptions we have to impose on the curvature function. We have to add one assumption compared to [4, Thm. 1.2].

Assumption 1.1. Let

$$
\Gamma_{+}=\left\{\left(\kappa_{i}\right) \in \mathbb{R}^{n}: \kappa_{i}>0 \quad \forall i=1, \ldots, n\right\} .
$$

Assume $F \in C^{\infty}\left(\Gamma_{+}\right) \cap C^{0}\left(\bar{\Gamma}_{+}\right)$to be a

- symmetric

- monotone

- homogeneous of degree 1

- concave

curvature function satisfying

$$
F_{\mid \partial \Gamma_{+}}=0
$$

the normalization

$$
F(1, \ldots, 1)=n
$$

and

$$
\frac{\partial F}{\partial \kappa_{i}} \kappa_{i} \geq \epsilon_{0} F \quad \forall i=1, \ldots, n
$$

for some $0<\epsilon_{0}(F) \leq \frac{1}{n}$.

We will prove the following result.

Theorem 1.1. Let $n \geq 2$ and

$$
x_{0}: M \hookrightarrow \mathbb{H}^{n+1}
$$

be the smooth embedding of a closed and strictly convex hypersurface $M_{0}$. Let F satisfy Assumption 1.1 and

$$
1<p \leq p_{0}:=\frac{1}{1-\epsilon_{0}} .
$$

Then the following statements hold.

(i) There exists a unique global solution

$$
x:[0, \infty) \times M \hookrightarrow \mathbb{H}^{n+1}
$$

of the curvature flow equation

$$
\begin{aligned}
\dot{x} & =\frac{1}{F^{p}} v \\
x(0, M) & =M_{0},
\end{aligned}
$$

where $v$ is the outward normal to the flow hypersurfaces $M_{t}=x(t, M)$ and $F$ is evaluated at the principal curvatures of $M_{t}$.

(ii) Representing $M_{0}$ as a graph in geodesic polar coordinates,

$$
M_{0}=\left\{\left(u\left(0, x^{i}\right), x^{i}(0, \xi)\right): \xi \in M\right\},
$$

where $u=x^{0}$ describes the radial distance to a point inside the convex body enclosed by $M_{0}$, we obtain that all flow hypersurfaces $M_{t}$ have a similar representation by a scalar function

$$
u:[0, \infty) \times \mathbb{S}^{n} \rightarrow \mathbb{R} .
$$

The rescaled hypersurfaces $\tilde{M}_{t}$ which are described via the scalar function

$$
\tilde{u}=u-\frac{t}{n^{p}}
$$

converge to a well-defined hypersurface in $C^{\infty}$. 
(iii) In case

$$
F=n K^{\frac{1}{n}}
$$

where $\mathrm{K}$ is the Gaussian curvature, we have

$$
p_{0}=\frac{n}{n-1} .
$$

Remark 1.1. Note that the convergence of the functions (14) can not be improved in general. A counterexample was recently given by Hung and Wang in case of the inverse mean curvature flow, $F=H$ and $p=1$, cf. [3]. Also in case $p>1$ we do not expect that the convergence can be improved, since the evolution equation of the traceless second fundamental form

$$
\|\AA\|^{2}=\|A\|^{2}-\frac{1}{n} H^{2}
$$

has the same structure in the first order terms as in case $p=1$.

Remark 1.2. Note that the $n$-th root of the Gaussian curvature

$$
F=n K^{\frac{1}{n}}
$$

fulfills Assumption 1.1 with $\epsilon_{0}=\frac{1}{n}$. To see that the set of curvature functions satisfying Assumption 1.1 contains considerably more functions the reader is referred to [2, Prop. 2.2.18]. In case $n=2$ this class contains, up to normalization and smoothness, all curvature functions of class $\left(K^{\star}\right)$ which are homogeneous of degree 1. For a definition of the class $\left(K^{\star}\right)$ compare [2, Sec. 2.2]. For arbitrary $n \geq 2$ it contains those 1-homogeneous functions which can be written as

$$
F=G K^{a}, a>0,
$$

where $G$ shares all the properties of a function belonging to class $(K)$ besides vanishing on $\partial \Gamma_{+}$.

Furthermore note that the value $\epsilon_{0}$ in (7) can not be larger than $\frac{1}{n}$, due to the homogeneity, which implies

$$
F^{i} \kappa_{i}=F
$$

\section{Notation}

Let us refresh some notation already used in [4]. We consider hypersurfaces in $\mathbb{H}^{n+1}$, the metric of which reads in geodesic polar coordinates

$$
d \bar{s}^{2}=d r^{2}+\vartheta^{2}(r) \sigma_{i j} d x^{i} d x^{j}
$$

where

$$
\vartheta(r)=\sinh (r),
$$

$r=x^{0}$ denotes the geodesic distance to some point $q \in \mathbb{H}^{n+1}$ and $\sigma_{i j}$ is the round metric of the $n$-sphere. In such coordinates, let the hypersurface $M$ be given as a graph over a geodesic sphere $\mathbb{S}^{n}$ around $q$,

$$
M=\left\{\left(u(x), x^{i}\right):\left(x^{i}\right) \in \mathbb{S}^{n}\right\}
$$

Then the induced metric of $M$ is given by

$$
g_{i j}=u_{i} u_{j}+\vartheta^{2}(u) \sigma_{i j}
$$

Let

$$
v^{2}=1+\vartheta^{-2} \sigma^{i j} u_{i} u_{j} \equiv 1+|D u|^{2},
$$

then the outward unit normal is given by

$$
\left(v^{\alpha}\right)=v^{-1}\left(1,-\vartheta^{-2} \sigma^{i j} u_{j}\right),
$$


$\alpha=0, \ldots, n$ and $i=1, \ldots, n$. For curvature functions depending on the second fundamental form and the metric,

$$
F=F\left(h_{k l}, g_{k l}\right),
$$

we let

$$
F^{k l}=\frac{\partial F}{\partial h_{k l}} .
$$

For functions $f$ defined on a manifold $M$, lower indices indicate covariant differentiation with respect to the induced metric of $M$, e.g. $u_{i}$ or $v_{i j}$.

A dot over a function or a tensor always indicates time derivation, e.g.

$$
\dot{v}=\frac{d}{d t} v,
$$

whereas a prime always denotes derivation with respect to a direct argument. For example, if $f=f(u)$, then

$$
f^{\prime}=\frac{d}{d u} f
$$

and

$$
\dot{f}=f^{\prime} \dot{u} .
$$

Note that this notation partially deviates from those in [1] and [2].

\section{Rough outline of the proof}

Let us shortly explain, which ingredient of our proof is the crucial one compared to the proof in [4]. In [4, Sec. 3] we proved the longtime existence of the solution to (1) by successively proving the existence of spherical barriers and of bounds on $v$ and the second fundamental form. This part of the proof is not affected by our relaxed assumptions at all, compare [4, Thm. 1.2 (1)]. Thus we have a longtime graph representation of the flow hypersurfaces,

$$
M_{t}=\left\{\left(u\left(t, x^{i}\right), x^{i}(t, \xi)\right): \xi \in M\right\} .
$$

In order to derive convergence of the flow, we started the decay estimates by proving that the quantity $v$ converges to 1 exponentially fast. In case $p>1$ this was only possible if $v$ was sufficiently small initially, due to the positively signed second order term in [4, equ. (4.3)]. As we will show in section 4, this restriction is not necessary in the situations described in Theorem 1.1. It turned out to be possible to further exploit the first negatively signed term in [4, equ. (4.3)] by using a better suited arrangement of the terms involved. We will be able to exploit this extra term with the help of the additional assumptions in Assumption 1.1 to derive exponential decay of $v-1$.

In order to obtain the optimal gradient estimate

$$
v-1 \leq c e^{-\frac{2 t}{n^{p}}}
$$

and to conclude the final convergence result we observe that the further arguments in [4] do not depend on the pinching restriction at all and the proofs of the further decay estimates apply literally, once one has proven decay of $v-1$.

\section{Proof of the main theorem}

As mentioned in section 3 it suffices to prove decay of $v-1$. The following proposition holds. 
Proposition 4.1. Let $x$ be the solution of (1) under Assumption 1.1 with strictly convex initial hypersurface $M_{0}$ and

$$
1<p \leq \frac{1}{1-\epsilon_{0}} .
$$

Let $u$ be a corresponding graph representation of the $M_{t}$. Then the quantity $v^{2}=1+|D u|^{2}$ satisfies the decay estimate

$$
v-1 \leq c e^{-\lambda t},
$$

with suitable positive constants $c, \lambda$ which depend on $n, M_{0}, p$ and $F$.

Proof. The flow (1) is of the form

$$
\dot{x}=-\Phi(F) \nu
$$

where

$$
\Phi(r)=-r^{-p}
$$

According to [1, equ. (5.28)] for such a flow we have

$$
\begin{aligned}
\dot{v}-\Phi^{\prime} F^{i j} v_{i j}= & -\Phi^{\prime} F^{i j} h_{i k} h_{j}^{k} v-2 v^{-1} \Phi^{\prime} F^{i j} v_{i} v_{j}+2 \Phi^{\prime} F^{i j} v_{i} u_{j} \frac{\bar{H}}{n} \\
& -\Phi^{\prime} F^{i j} g_{i j} \frac{\bar{H}^{2}}{n^{2}} v-\Phi^{\prime} F^{i j} u_{i} u_{j} \frac{\bar{H}^{\prime}}{n} v+\frac{\bar{H}}{n}\left(\Phi-\Phi^{\prime} F\right)|D u|^{2}+2 \Phi^{\prime} F \frac{\bar{H}}{n} v^{2} \\
= & -\Phi^{\prime} F^{i j}\left(h_{i k} h_{j}^{k}-2 \frac{\bar{H}}{n} h_{i j}+\frac{\bar{H}^{2}}{n^{2}} g_{i j}\right) v+\left(1-\frac{1}{p}\right) \Phi^{\prime} F \frac{\bar{H}}{n} v^{2} \\
& -2 \Phi^{\prime} F \frac{\bar{H}}{n} v+\left(1+\frac{1}{p}\right) \Phi^{\prime} F \frac{\bar{H}}{n}-\Phi^{\prime} F^{i j} u_{i} u_{j} \frac{\bar{H}^{\prime}}{n} v \\
& -2 v^{-1} \Phi^{\prime} F^{i j} v_{i} v_{j}+2 \Phi^{\prime} F^{i j} v_{i} u_{j} \frac{\bar{H}}{n}
\end{aligned}
$$

Define

$$
w=(v-1) e^{\lambda t}, \quad \lambda>0,
$$

and suppose for $0<T<\infty$ that

$$
\sup _{[0, T] \times M} w=w\left(t_{0}, \xi_{0}\right) \geq 1, \quad t_{0}>0 .
$$

Due to $\left[1\right.$, equ. (5.29)] we have at $\left(t_{0}, \xi_{0}\right)$

$$
0=v_{i}=-v^{2} h_{i}^{k} u_{k}+v \frac{\bar{H}}{n} u_{i} .
$$

Choose Riemannian normal coordinates around $\left(t_{0}, \xi_{0}\right)$, in which

$$
g_{i j}=\delta_{i j}, \quad h_{i j}=\kappa_{i} \delta_{i j}
$$

Since $v\left(t_{0}, \xi_{0}\right)>1$, there exists an index $j \in\{1, \ldots, n\}$, such that $u_{j} \neq 0$ and thus

$$
\kappa_{j}=v^{-1} \frac{\bar{H}}{n} .
$$

Noting that in the hyperbolic space the mean curvature $\bar{H}$ of the slices $\left\{x^{0}=u\right\}$ satisfies

$$
\frac{\bar{H}}{n}=\operatorname{coth} u
$$

that

$$
\frac{\partial F}{\partial \kappa_{i}} \geq \epsilon_{0} \frac{F}{\kappa_{i}} \quad \forall 1 \leq i \leq n
$$

and that in our present coordinate system we have

$$
F^{i i}=\frac{\partial F}{\partial \kappa_{i}},
$$


cf. [2, Lemma 2.1.9], we obtain from (38) at the point $\left(t_{0}, \xi_{0}\right)$ that

$$
\begin{aligned}
0 \leq & \lambda w-\Phi^{\prime} F^{j j} \frac{\bar{H}^{2}}{n^{2}} \frac{(v-1)^{2}}{v} e^{\lambda t_{0}}+\left(1-\frac{1}{p}\right) \Phi^{\prime} F \frac{\bar{H}}{n} v^{2} e^{\lambda t_{0}} \\
& -2 \Phi^{\prime} F \frac{\bar{H}}{n} v e^{\lambda t_{0}}+\left(1+\frac{1}{p}\right) \Phi^{\prime} F \frac{\bar{H}}{n} e^{\lambda t_{0}}-\Phi^{\prime} F^{i j} u_{i} u_{j}\left(1-\frac{\bar{H}^{2}}{n^{2}}\right) v e^{\lambda t_{0}} \\
\leq & \lambda w-\epsilon_{0} \Phi^{\prime} F \frac{\bar{H}}{n}(v-1)^{2} e^{\lambda t_{0}}+\left(1-\frac{1}{p}\right) \Phi^{\prime} F \frac{\bar{H}}{n} v^{2} e^{\lambda t_{0}} \\
& -2 \Phi^{\prime} F \frac{\bar{H}}{n} v e^{\lambda t_{0}}+\left(1+\frac{1}{p}\right) \Phi^{\prime} F \frac{\bar{H}}{n} e^{\lambda t_{0}}-\Phi^{\prime} F^{i j} u_{i} u_{j}\left(1-\frac{\bar{H}^{2}}{n^{2}}\right) v e^{\lambda t_{0}} \\
= & \lambda w+\left(1-\frac{1}{p}-\epsilon_{0}\right) \Phi^{\prime} F \frac{\bar{H}}{n} v^{2} e^{\lambda t_{0}}-\left(2-2 \epsilon_{0}\right) \Phi^{\prime} F \frac{\bar{H}}{n} v e^{\lambda t_{0}} \\
& +\left(1+\frac{1}{p}-\epsilon_{0}\right) \Phi^{\prime} F \frac{\bar{H}}{n} e^{\lambda t_{0}}+c e^{\left(\lambda-\frac{2}{n^{p}}\right) t_{0}} \\
\leq & \lambda w-\left(1-\epsilon_{0}\right) \Phi^{\prime} F \frac{\bar{H}}{n} w+\left(1-\frac{1}{p}-\epsilon_{0}\right) \Phi^{\prime} F \frac{\bar{H}}{n} v w+c e^{\left(\lambda-\frac{2}{n^{p}}\right) t_{0}} \\
& <0
\end{aligned}
$$

for small $\lambda$ and large $t_{0}$, where we also used

$$
\begin{gathered}
F+F^{-1}+v \leq c, \\
\frac{\bar{H}}{n}-1 \leq c e^{-\frac{2 t}{n^{p}}}
\end{gathered}
$$

and that the $\kappa_{i}$ range in a compact set of $\Gamma_{+}$. We derived those facts in [4, Cor. 3.6, Lemma 3.7, Prop. 3.10 and Prop. 3.11]. This is a contradiction and thus $w$ is bounded.

Remark 4.1. In case of the Gaussian curvature

$$
F=n K^{\frac{1}{n}}
$$

we obtain

$$
\frac{1}{1-\epsilon_{0}}=\frac{n}{n-1}
$$

which proves part (iii) of Theorem 1.1.

\section{References}

[1] Claus Gerhardt. Closed Weingarten hypersurfaces in space forms. Geom. Anal. Calc. Var., pages 71-98, 1996.

[2] Claus Gerhardt. Curvature problems, volume 39 of Series in Geometry and Topology. International Press of Boston Inc., 2006.

[3] Pei-Ken Hung and Mu Tao Wang. Inverse mean curvature flows in the hyperbolic 3-space revisited. Calc. Var. Partial Differential Equations, 2014. doi: 10.1007/s00526-014-0780-3.

[4] Julian Scheuer. Non-scale-invariant inverse curvature flows in hyperbolic space. Calc. Var. Partial Differential Equations, 2014. doi: 10.1007/s00526-014-0742-9. 